\title{
EXISTENCE RESULTS FOR POSITIVE SOLUTIONS \\ OF NON-HOMOGENEOUS BVPS FOR \\ SECOND ORDER DIFFERENCE EQUATIONS WITH \\ ONE-DIMENSIONAL $p$-LAPLACIAN
}

\author{
YUJi LiU
}

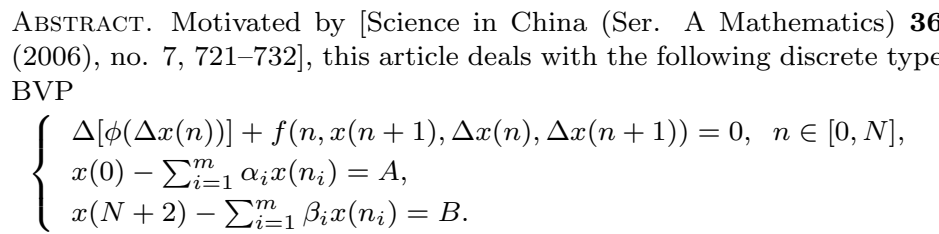

The sufficient conditions to guarantee the existence of at least three positive solutions of the above multi-point boundary value problem are established by using a new fixed point theorem obtained in [5]. An example is presented to illustrate the main result. It is the purpose of this paper to show that the approach to get positive solutions of BVPs by using multifixed-point theorems can be extended to treat nonhomogeneous BVPs. The emphasis is put on the nonlinear term $f$ involved with the first order delta operator $\Delta x(n)$.

\section{Introduction}

In recent years, there has been a large number of papers interested in proving the existence of positive solutions of the boundary value problems (BVPs for short) of the difference equations since these BVPs have extensive applications, see the papers [1-9] and the references therein. We now discuss briefly several of the appropriate papers on the topic.

In the recent paper, the authors in [7] investigated the following BVPs for the third-order nonlinear difference equation

$$
\Delta^{3} x(n-2)=a(n) f(x(n)), \quad n \in[2, T+2],
$$

Received April 15, 2008.

2000 Mathematics Subject Classification. 34B10, 34B15, 35B10.

Key words and phrases. one-dimension p-Laplacian difference equation, multi-point boundary value problem, positive solution.

Supported by Natural Science Foundation of Hunan province (No:06JJ5008) and Natural Science Foundation of Guangdong Province (No:7004569). 
satisfying several kinds of different boundary conditions. The Green functions were constructed and their bounds are evaluated. Some verifiable conditions for the existence of positive solutions of the corresponding BVPs were established.

In paper [12], the discrete BVP of the third-order difference equation

$$
\left\{\begin{array}{l}
\Delta^{3} x(t)=f(t, x(t+1)), \quad t_{1} \leq t \leq t_{3}-1 \\
x\left(t_{1}\right)=0 \\
\alpha x\left(t_{2}\right)-\beta \Delta^{2} x\left(t_{2}\right)=0 \\
\gamma x\left(t_{3}\right)+\delta \Delta^{3} x\left(t_{3}\right)=0
\end{array}\right.
$$

and the eigenvalue problem

$$
\Delta^{3} x(t)=\lambda f(t, x(t+1))
$$

with the same boundary conditions, where $t_{1}<t_{2}<t_{3}$ are distinct integers, were discussed. Under various assumptions on $f$ and $\lambda$, the existence of positive solutions of both problems were proved by applying a fixed point theorem.

In [5], a new fixed-point theorem of functional type in the cone is established. By using this fixed-point theorem and imposing growth conditions on the nonlinearity, the existence of three positive solutions for the boundary value problem

$$
\left\{\begin{array}{l}
{\left[\phi\left(x^{\prime}(t)\right]^{\prime}+q(t) f\left(t, x(t), x^{\prime}(t)\right)=0, \quad t \in(0,1)\right.} \\
x(0)=x(1)=0 \text { or } x(0)=x^{\prime}(1)=0 .
\end{array}\right.
$$

The discrete analogue of above BVP is as follows:

$$
\left\{\begin{array}{l}
\Delta[\phi(\Delta x(n))]+f(n, x(n), \Delta x(n))=0, \quad n \in[0, T] \\
x(0)=x(T+2)=0 \text { or } x(0)=\Delta x(T+1)=0 .
\end{array}\right.
$$

The authors in $[2,8,15]$ establish existence results for positive solutions of its special case

$$
\left\{\begin{array}{l}
\Delta^{2} x(n)+f(n, x(n))=0, n \in[0, T], \\
x(0)=x(T+2)=0 \text { or } x(0)=\Delta x(T+1)=0 .
\end{array}\right.
$$

In the known papers, the explicit expressions of the associated Green's functions are used to get multiple positive solutions of the two point or three point BVPs of difference equations by using the Leggett-Williams type fixed-point theorems, see $[1,2,3,4,5,6,8,9,11,14,15,18]$ and the references therein.

It is well known that the following BVP

$$
\left\{\begin{array}{l}
\Delta(p(n) \Delta x(n-1))+q(n) x(n)=f(n, x(n)), n \in[1, T], \\
x(0)=A, \\
x(T+1)=B
\end{array}\right.
$$

is called generalized discrete BVP of Emden-Fowler equation [17]. Let

$$
F\left(u_{1}, \ldots, u_{T}\right)=\sum_{k=1}^{T} \int_{0}^{u_{k}} f(k, s) d s, \quad c(n)=q(n)-p(n)-p(n+1)
$$


and

$$
M=\left(\begin{array}{cccccc}
c(1) & p(2) & 0 & \ldots & 0 & 0 \\
p(2) & c(2) & p(3) & \ldots & 0 & 0 \\
0 & p(3) & c(3) & \ldots & 0 & 0 \\
\cdot & \cdot & \cdot & \cdots & . & \cdot \\
\cdot & . & . & \cdots & . & \cdot \\
\cdot & . & . & \ldots & . & \cdot \\
0 & 0 & 0 & \cdots & c(T-1) & p(T) \\
0 & 0 & 0 & \ldots & p(T) & c(T)
\end{array}\right)
$$

By using the critical point theory, it was proved that $\operatorname{BVP}(2)$ has at least one solutions if one of the following conditions is satisfied:

$f_{1}: M$ is positive or negative, and $F$ satisfies that there exist constants $a_{1}>0, a_{2}>0, R>0$ and $1<\alpha<2$ such that

$$
F\left(u_{1}, \ldots, u_{T}\right) \leq a_{1}\left\|\left(u_{1}, \ldots, u_{T}\right)\right\|^{\alpha}+a_{2} \text { for }\left\|\left(u_{1}, \ldots, u_{T}\right)\right\|>R ;
$$

$\boldsymbol{f}_{\mathbf{2}}: M$ is no singular and there exists a constant $c_{0}>0$ such that $f(n, x) \leq$ $c_{0}$ for every $n \in[1, T]$ and $x \in \mathbb{R}$;

$f_{\mathbf{3}}: F$ satisfies that there exist constants $a_{1}>0, a_{2}>0, R>0$ and $\beta>2$ such that

$$
F\left(u_{1}, \ldots, u_{T}\right) \geq a_{1}\left\|\left(u_{1}, \ldots, u_{T}\right)\right\|^{\alpha}+a_{2} \text { for }\left\|\left(u_{1}, \ldots, u_{T}\right)\right\| \geq R .
$$

It is easy to see that $\operatorname{BVP}(2)$ can be transformed to the following BVP:

$$
\begin{cases}\Delta^{2} x(n-1)= & \left(\frac{p(n)-q(n)}{p(n+1)}-1\right) x(n)+\left(1-\frac{p(n)}{p(n+1)}\right) x(n-1) \\ & \quad+\frac{f(n, x(n))}{p(n+1)}, n \in[1, T], \\ x(0)=A, & \\ x(T+1)=B . & \end{cases}
$$

However, in applications, we find that the nonhomogeneous BVP

$$
\left\{\begin{array}{l}
\Delta^{2} x(n-1)=-4, \quad n \in[0, T], \\
x(0)=A, \\
x(T+1)=B
\end{array}\right.
$$

has unique positive solution

$$
x(n)=\frac{n B+(T+1-n) A}{T+1}+2 T n-2(n-1) n \text { if } A \geq 0 \text { and } B \geq 0 .
$$

While the following nonhomogeneous multi-point BVP

$$
\left\{\begin{array}{l}
\Delta^{2} x(n-1)=-4, \quad n \in[0, T], T>24, \\
x(0)=\frac{1}{2} x(30)+A \\
x(T+1)=\frac{1}{2} x(80)+B
\end{array}\right.
$$


has unique solution

$$
\begin{aligned}
x(n)= & -2(n-1) n+\frac{B-A+2 T^{2}+2 T-5450}{T-24} n \\
& +\frac{2 T A-78 A+30 B+60 T^{2}-1680 T-121740}{T-24} .
\end{aligned}
$$

Since

$$
x(T+1)=\frac{T A+T B-79 A+31 B+110 T^{2}-7080 T-127190}{T-24},
$$

we find that $\operatorname{BVP}(* *)$ has no positive solution if

$A \geq 0, B \geq 0, T A+T B-79 A+31 B+110 T^{2}-7080 T-127190<0$.

To the best of knowledge, there exists no paper discussed the existence of multiple positive solutions of multi-point BVPs of $p$-Laplacian difference equations whose nonlinear term is dependent on $\Delta x(n+1)$. To apply fixed point theorem in cones in Banach spaces, the difficult to study this kind of BVP comes from that it is not easy to establish the associated Green's functions. To use critical point theory to get solutions of this kind of BVP comes from that it is not easy to construct the functionals.

Motivated by the reason and papers mentioned above, the purpose of this paper is to investigate the more generalized BVPs for the second order $p$ Laplacian difference equation with multi-point BCs, i.e., the BVP

$$
\left\{\begin{array}{l}
\Delta[\phi(\Delta x(n))]+f(n, x(n+1), \Delta x(n), \Delta x(n+1))=0, n \in[0, N], \\
x(0)-\sum_{i=1}^{m} \alpha_{i} x\left(n_{i}\right)=A, \\
x(N+2)-\sum_{i=1}^{m} \beta_{i} x\left(n_{i}\right)=B
\end{array}\right.
$$

where

- $N>1$ an integer, $0<n_{1}<\cdots<n_{m}<N+2$;

- $\alpha_{i}, \beta_{i} \geq 0$ for all $i=1, \ldots, m, A \geq 0, B \geq 0$;

- $f$ is continuous and positive;

- $\phi$ is called $p$-Laplacian, $\phi(x)=|x|^{p-2} x$ for $x \neq 0$ and $\phi(0)=0$ with $p>1$, its inverse function is denoted by $\phi^{-1}(x)$ with $\phi^{-1}(x)=|x|^{q-2} x$ for $x \neq 0$ and $\phi^{-1}(0)=0$ with $1 / p+1 / q=1$.

- $\sum_{i=a}^{b} x(i)=0$ if $a, b \in \mathbb{Z}$ and $b<a$, where $\mathbb{Z}$ is the integer set, denote $[a, b]=\{a, a+1, \ldots, b\}$ for $a, b \in \mathbb{Z}$ with $a \leq b$.

We establish sufficient conditions for the existence of at least three positive solutions of $\operatorname{BVP}(3)$. The methods and the results in this paper, which are not based upon the Green's functions, are different from those in known papers.

The remainder of this paper is organized as follows: to get the main results, in Section 2, we first give eleven lemmas, then Theorem L1 and Theorem L2 and their proofs are presented at the end of this section, and an example is given in Section 3 to illustrate the main results. 


\section{Main results}

In this section, we first present some background definitions in Banach spaces and state the important three fixed point theorem and lemmas. Then the main results are given and proved.

Definition 2.1. Let $X$ be a semi-ordered real Banach space. The nonempty convex closed subset $P$ of $X$ is called a cone in $X$ if

(i) $a x \in P$ for all $x \in P$ and $a \geq 0$;

(ii) $x \in X$ and $-x \in X$ imply $x=0$.

Definition 2.2. A map $\psi: P \rightarrow[0,+\infty)$ is a nonnegative continuous concave or convex functional map provided $\psi$ is nonnegative, continuous and satisfies

$$
\psi(t x+(1-t) y) \geq t \psi(x)+(1-t) \psi(y),
$$

or

$$
\psi(t x+(1-t) y) \leq t \psi(x)+(1-t) \psi(y)
$$

for all $x, y \in P$ and $t \in[0,1]$.

Definition 2.3. An operator $T: X \rightarrow X$ is completely continuous if it is continuous and maps bounded sets into pre-compact sets.

Definition 2.4. Let $r>a>0, L>0$ be positive constants, $\psi$ be a nonnegative continuous concave functional on the cone $P, \alpha$ and $\beta$ be two nonnegative continuous convex functional on the cone $P$. Define the convex sets as follows:

$$
\begin{aligned}
& P(\alpha, r ; \beta, L)=\{x \in P: \alpha(x)<r, \beta(x)<L\}, \\
& \bar{P}(\alpha, r ; \beta, L)=\{x \in P: \alpha(x) \leq r, \beta(x) \leq L\}, \\
& P(\alpha, r ; \beta, L ; \psi, a)=\{x \in P: \alpha(x)<r, \beta(x)<L, \psi(x)>a\}, \\
& \bar{P}(\alpha, r ; \beta, L ; \psi, a)=\{x \in P: \alpha(x) \leq r, \beta(x) \leq L, \psi(x) \geq a\} .
\end{aligned}
$$

Suppose that the nonnegative continuous convex functional on the cone $P$ $\alpha$ and $\beta$ satisfy the followings:

(a) there exists a constant $M>0$ such that $\|x\| \leq \max \{\alpha(x), \beta(x)\} M$ for all $x \in P$;

(b) $P(\alpha, r ; \beta, L) \neq \emptyset$ for each $r>0, L>0$.

Next, we state fixed-point theorems.

Lemma 2.1 ([5]). Let $X$ be a real Banach space, $P$ be a cone in $X, r_{2}>$ $d>b>r_{1}>0, L_{2}>L_{1}>0$ be constants, $\alpha$ and $\beta$ be two nonnegative continuous convex functionals on the cone $P$ satisfying (a) and (b), $\psi$ be a nonnegative continuous concave functional on the cone $P$ with $\psi(x) \leq \alpha(x)$ for each $x \in \bar{P}\left(\alpha, r_{2} ; \beta, L_{2}\right)$. Let $T: \bar{P}\left(\alpha, r_{2} ; \beta, L_{2}\right) \rightarrow \bar{P}\left(\alpha, r_{2} ; \beta, L_{2}\right)$ be a completely continuous operator. Suppose

$\left(C_{1}\right)\left\{y \in \bar{P}\left(\alpha, d ; \beta, L_{2} ; \psi, b\right) \mid \psi(y)>b\right\} \neq \emptyset$ and $\psi(T y)>b$ for every $y \in$ $\bar{P}\left(\alpha, d ; \beta, L_{2} ; \psi, b\right)$

$\left(C_{2}\right) \alpha(T y)<r_{1}$ and $\beta(T y)<L_{1}$ for every $y \in \bar{P}\left(\alpha, r_{1} ; \beta, L_{1}\right)$; 
$\left(C_{3}\right) \psi(T y)>b$ for $y \in \bar{P}\left(\alpha, r_{2} ; \beta, L_{2} ; \psi, b\right)$ with $\alpha(T y)>d$.

Then $T$ has at least three fixed points $y_{1}, y_{2}$ and $y_{3}$ such that

$$
\begin{aligned}
& y_{1} \in P\left(\alpha, r_{1} ; \beta, L_{1}\right), \\
& y_{2} \in\left\{y \in \bar{P}\left(\alpha, r_{2} ; \beta, L_{2} ; \psi, b\right), \psi(y)>b\right\}, \\
& y_{3} \in \bar{P}\left(\alpha, r_{2} ; \beta, L_{2}\right) \backslash\left[\bar{P}\left(\alpha, r_{2} ; \beta, L_{2} ; \psi, b\right) \sqcup \bar{P}\left(\alpha, r_{1} ; \beta, L_{1}\right)\right] .
\end{aligned}
$$

The following assumptions, which will be used in the proofs of all lemmas, are supposed.

$\left(H_{1}\right) f:[0, N] \times[0,+\infty) \times \mathbb{R}^{2} \rightarrow(0,+\infty)$ is continuous.

$\left(H_{2}\right) A \geq 0, B \geq 0, \alpha_{i} \geq 0, \beta_{i} \geq 0$ satisfy $\sum_{i=1}^{m} \alpha_{i}<1$ and $\sum_{i=1}^{m} \beta_{i} \leq 1$ with

$$
B\left(1-\sum_{i=1}^{m} \alpha_{i}\right) \neq A\left(1-\sum_{i=1}^{m} \beta_{i}\right) .
$$

Choose $X=\mathbb{R}^{N+3}$. We call $x \leq y$ for $x, y \in X$ if $x(n) \leq y(n)$ for all $n \in[0, N+2]$, define the norm

$$
\|x\|=\max \left\{\max _{n \in[0, N+2]}|x(n)|, \max _{n \in[0, N+1]}|\Delta x(n)|\right\} .
$$

It is easy to see that $X$ is a semi-ordered real Banach space.

Choose

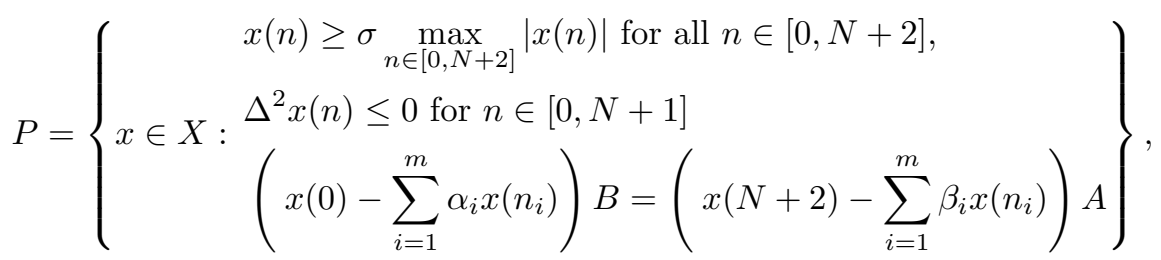

where $\sigma=\min \left\{\frac{n}{N+2}, \frac{N+2-n}{N+2}\right\}$. Then $P$ is a cone in $X$.

Choose $\left[\frac{N+2}{2}\right]>k>0$, where $[x]$ denotes the integer not greater than $x$. Define the functionals on $P: P \rightarrow[0,+\infty)$ by

$$
\begin{aligned}
\alpha(x) & =\max _{n \in[0, N+2]}|x(n)|, \\
\beta(x) & =\max _{n \in[0, N+1]}|\Delta x(n)|,
\end{aligned}
$$

and

$$
\psi(x)=\min _{t \in[k, N+2-k]}|x(n)| .
$$

For $x \in P$, we have that

$$
\begin{aligned}
|x(0)| & =\left|\frac{\frac{A}{B} x(N+2)+\sum_{i=1}^{m} \alpha_{i} x\left(n_{i}\right)-\frac{A}{B} \sum_{i=1}^{m} \beta_{i} x\left(n_{i}\right)-\left(\frac{A}{B}+\sum_{i=1}^{m} \alpha_{i}-\frac{A}{B} \sum_{i=1}^{m} \beta_{i}\right) x(0)}{1-\frac{A}{B}-\sum_{i=1}^{m} \alpha_{i}+\frac{A}{B} \sum_{i=1}^{m} \beta_{i}}\right| \\
& \leq \frac{\frac{A}{B} \sum_{i=0}^{N+1}|\Delta x(s)|+\sum_{i=1}^{m}\left|\alpha_{i}-\frac{A}{B} \beta_{i}\right| \sum_{i=0}^{n_{i}-1}|\Delta x(s)|}{\left|1-\frac{A}{B}-\sum_{i=1}^{m} \alpha_{i}+\frac{A}{B} \sum_{i=1}^{m} \beta_{i}\right|}
\end{aligned}
$$




$$
\leq \frac{\frac{A}{B}(N+2)+\sum_{i=1}^{m}\left|\alpha_{i}-\frac{A}{B} \beta_{i}\right| n_{i}}{\left|1-\frac{A}{B}-\sum_{i=1}^{m} \alpha_{i}+\frac{A}{B} \sum_{i=1}^{m} \beta_{i}\right|} \max _{n \in[0, N+1]}|\Delta x(n)| .
$$

It follows that

$$
\begin{aligned}
|x(n)| & \leq|x(n)-x(0)|+|x(0)| \\
& \leq\left(N+2+\frac{\frac{A}{B}(N+2)+\sum_{i=1}^{m}\left|\alpha_{i}-\frac{A}{B} \beta_{i}\right| n_{i}}{\left|1-\frac{A}{B}-\sum_{i=1}^{m} \alpha_{i}+\frac{A}{B} \sum_{i=1}^{m} \beta_{i}\right|}\right) \max _{n \in[0, N+1]}|\Delta x(n)| .
\end{aligned}
$$

Then

$$
\alpha(x) \leq\left(N+2+\frac{\frac{A}{B}(N+2)+\sum_{i=1}^{m}\left|\alpha_{i}-\frac{A}{B} \beta_{i}\right| n_{i}}{\left|1-\frac{A}{B}-\sum_{i=1}^{m} \alpha_{i}+\frac{A}{B} \sum_{i=1}^{m} \beta_{i}\right|}\right) \beta(x), x \in P .
$$

So there exists a constant $M>0$ such that $\|x\| \leq \max \{\alpha(x), \beta(x)\}$ for all $x \in P$. It is easy to see that $\alpha$ and $\beta$ are two nonnegative continuous convex functional on the cone $P$ satisfying $(a)$ and $(b)$ and $\psi$ is a nonnegative continuous concave functional on the cone $P$ with $\psi(x) \leq \alpha(x)$ for each $x \in P$.

For $x \in P$, consider the following BVP

$$
\left\{\begin{array}{l}
\Delta[\phi(\Delta y(n))]+f(n, x(n+1), \Delta x(n), \Delta x(n+1))=0, n \in[0, N], \\
y(0)-\sum_{i=1}^{m} \alpha_{i} y\left(n_{i}\right)=A, \\
y(N+2)-\sum_{i=1}^{m} \beta_{i} y\left(n_{i}\right)=B .
\end{array}\right.
$$

Lemma 2.2. Suppose that

$\left(H_{3}\right) \frac{1-\sum_{i=1}^{m} \beta_{i}}{1-\sum_{i=1}^{m} \alpha_{i}} A-B>0$, there exists a sequence $q$ such that $f(n, x, u, v) \geq$ $q(n)$ for all $n \in[0, N], x, u, v \in \mathbb{R}$ and

$$
\begin{aligned}
& -\frac{1-\sum_{i=1}^{m} \beta_{i}}{1-\sum_{i=1}^{m} \alpha_{i}}\left(\sum_{i=1}^{m} \alpha_{i} \sum_{j=0}^{n_{i}-1} \phi^{-1}\left(\sum_{s=0}^{j-1} q(s)\right)\right)-\left(1-\sum_{i=1}^{m} \beta_{i}\right) \sum_{i=0}^{N+1} \phi^{-1}\left(\sum_{s=0}^{i-1} q(s)\right) \\
& -\sum_{i=1}^{m} \beta_{i} \sum_{j=n_{i}}^{N+1} \phi^{-1}\left(\sum_{s=0}^{j-1} q(s)\right)+\frac{1-\sum_{i=1}^{m} \beta_{i}}{1-\sum_{i=1}^{m} \alpha_{i}} A-B<0
\end{aligned}
$$

Furthermore, suppose that $\left(H_{1}\right)$ and $\left(H_{2}\right)$ hold. If $y$ is a solution of BVP (4), then there exits unique $n_{0} \in[0, N]$ such that $\Delta y\left(n_{0}\right)>0$ and $\Delta y\left(n_{0}+1\right) \leq 0$.

Proof. Suppose $y$ satisfies (4). It follows from (4) that

$$
y(n)=y(0)+\sum_{i=0}^{n-1} \phi^{-1}\left(\phi(\Delta y(0))-\sum_{s=0}^{i-1} f(s, x(s+1), \Delta x(s), \Delta x(s+1))\right) .
$$

The BCs in (4) imply that

$$
y(0)=y(0) \sum_{i=1}^{m} \alpha_{i}+\sum_{i=1}^{m} \alpha_{i} \sum_{j=0}^{n_{i}-1} \phi^{-1}\left(\phi(\Delta y(0))-\sum_{s=0}^{j-1} f(s, x(s+1), \Delta x(s), \Delta x(s+1))\right)+A
$$


and

$$
\begin{aligned}
& y(0)+\sum_{i=0}^{N+1} \phi^{-1}\left(\phi(\Delta y(0))-\sum_{s=0}^{i-1} f(s, x(s+1), \Delta x(s), \Delta x(s+1))\right) \\
= & y(0) \sum_{i=1}^{m} \beta_{i}+\sum_{i=1}^{m} \beta_{i} \sum_{j=0}^{n_{i}-1} \phi^{-1}\left(\phi(\Delta y(0))-\sum_{s=0}^{j-1} f(s, x(s+1), \Delta x(s), \Delta x(s+1))\right)+B .
\end{aligned}
$$

It follows that

$$
y(0)=\frac{1}{1-\sum_{i=1}^{m} \alpha_{i}}\left(\sum_{i=1}^{m} \alpha_{i} \sum_{j=0}^{n_{i}-1} \phi^{-1}\left(\phi(\Delta y(0))-\sum_{s=0}^{i-1} f(s, x(s+1), \Delta x(s), \Delta x(s+1))\right)+A\right)
$$

and

$$
\begin{aligned}
& \frac{1-\sum_{i=1}^{m} \beta_{i}}{1-\sum_{i=1}^{m} \alpha_{i}}\left(\sum_{i=1}^{m} \alpha_{i} \sum_{j=0}^{n_{i}-1} \phi^{-1}\left(\phi(\Delta y(0))-\sum_{s=0}^{j-1} f(s, x(s+1), \Delta x(s), \Delta x(s+1))\right)+A\right) \\
& +\sum_{i=0}^{N+1} \phi^{-1}\left(\phi(\Delta y(0))-\sum_{s=0}^{i-1} f(s, x(s+1), \Delta x(s), \Delta x(s+1))\right) \\
= & \sum_{i=1}^{m} \beta_{i} \sum_{j=0}^{n_{i}-1} \phi^{-1}\left(\phi(\Delta y(0))-\sum_{s=0}^{j-1} f(s, x(s+1), \Delta x(s), \Delta x(s+1))\right)+B .
\end{aligned}
$$

Similarly, we can get that

(6) $y(n)=y(N+2)-\sum_{i=n}^{N+1} \phi^{-1}\left(\phi(\Delta y(N+1))+\sum_{s=i}^{N} f(s, x(s+1), \Delta x(s), \Delta x(s+1))\right)$.

The BCs in (4) imply that

$$
\begin{aligned}
& y(N+2) \\
= & x(N+2) \sum_{i=1}^{m} \beta_{i}-\sum_{i=1}^{m} \beta_{i} \sum_{j=n_{i}}^{N+1} \phi^{-1}\left(\phi(\Delta y(N+1))+\sum_{s=j}^{N} f(s, x(s+1), \Delta x(s), \Delta x(s+1))\right)+B,
\end{aligned}
$$

and

$$
\begin{aligned}
& y(N+2)-\sum_{i=0}^{N+1} \phi^{-1}\left(\phi(\Delta y(N+1))+\sum_{s=i}^{N} f(s, x(s+1), \Delta x(s), \Delta x(s+1))\right) \\
= & y(N+2) \sum_{i=1}^{m} \alpha_{i}-\sum_{i=1}^{m} \alpha_{i} \sum_{j=i}^{N+1} \phi^{-1}\left(\phi(\Delta y(N+1))+\sum_{s=j}^{N} f(s, x(s+1), \Delta x(s), \Delta x(s+1))\right)+A .
\end{aligned}
$$

Then

$$
\begin{aligned}
& \frac{1-\sum_{i=1}^{m} \beta_{i}}{1-\sum_{i=1}^{m} \alpha_{i}}\left[\sum_{i=0}^{N+1} \phi^{-1}\left(\phi(\Delta y(N+1))+\sum_{s=n}^{N} f(s, x(s+1), \Delta x(s), \Delta x(s+1))\right)\right. \\
& \left.-\sum_{i=1}^{m} \alpha_{i} \sum_{j=i}^{N+1} \phi^{-1}\left(\phi(\Delta y(N+1))+\sum_{s=j}^{N} f(s, x(s+1), \Delta x(s), \Delta x(s+1))\right)+A\right]
\end{aligned}
$$


$=-\sum_{i=1}^{m} \beta_{i} \sum_{j=n_{i}}^{N+1} \phi^{-1}\left(\phi(\Delta y(N+1))+\sum_{s=j}^{N} f(s, x(s+1), \Delta x(s), \Delta x(s+1))\right)+B$.

Step 1. Prove that $\phi(\Delta y(0)) \geq 0$. In fact, let

$$
\begin{aligned}
F(c)= & \frac{1-\sum_{i=1}^{m} \beta_{i}}{1-\sum_{i=1}^{m} \alpha_{i}}\left(\sum_{i=1}^{m} \alpha_{i} \sum_{j=0}^{n_{i}-1} \phi^{-1}\left(c-\sum_{s=0}^{j-1} f(s, x(s+1), \Delta x(s), \Delta x(s+1))\right)+A\right) \\
& +\sum_{i=0}^{N+1} \phi^{-1}\left(c-\sum_{s=0}^{i-1} f(s, x(s+1), \Delta x(s), \Delta x(s+1))\right) \\
& -\sum_{i=1}^{m} \beta_{i} \sum_{j=0}^{n_{i}-1} \phi^{-1}\left(c-\sum_{s=0}^{j-1} f(s, x(s+1), \Delta x(s), \Delta x(s+1))\right)-B .
\end{aligned}
$$

Then $F(\phi(\Delta y(0)))=0$ and

$$
\begin{aligned}
F(c)= & \frac{1-\sum_{i=1}^{m} \beta_{i}}{1-\sum_{i=1}^{m} \alpha_{i}}\left(\sum_{i=1}^{m} \alpha_{i} \sum_{j=0}^{n_{i}-1} \phi^{-1}\left(c-\sum_{s=0}^{j-1} f(s, x(s+1), \Delta x(s), \Delta x(s+1))\right)+A\right) \\
& +\left(1-\sum_{i=1}^{m} \beta_{i}\right) \sum_{i=0}^{N+1} \phi^{-1}\left(c-\sum_{s=0}^{i-1} f(s, x(s+1), \Delta x(s), \Delta x(s+1))\right) \\
& +\sum_{i=1}^{m} \beta_{i} \sum_{j=n_{i}}^{N+1} \phi^{-1}\left(c-\sum_{s=0}^{j-1} f(s, x(s+1), \Delta x(s), \Delta x(s+1))\right)-B .
\end{aligned}
$$

One sees from $\left(H_{1}\right)$ and $\left(H_{2}\right)$ that $F(c)$ is increasing on $(-\infty,+\infty) .\left(H_{1}\right)$ implies that

$$
\begin{aligned}
F(0)= & -\frac{1-\sum_{i=1}^{m} \beta_{i}}{1-\sum_{i=1}^{m} \alpha_{i}}\left(\sum_{i=1}^{m} \alpha_{i} \sum_{j=0}^{n_{i}-1} \phi^{-1}\left(\sum_{s=0}^{j-1} f(s, x(s+1), \Delta x(s), \Delta x(s+1))\right)\right) \\
& -\left(1-\sum_{i=1}^{m} \beta_{i}\right) \sum_{i=0}^{N+1} \phi^{-1}\left(\sum_{s=0}^{i-1} f(s, x(s+1), \Delta x(s), \Delta x(s+1))\right) \\
& -\sum_{i=1}^{m} \beta_{i} \sum_{j=n_{i}}^{N+1} \phi^{-1}\left(\sum_{s=0}^{j-1} f(s, x(s+1), \Delta x(s), \Delta x(s+1))\right) \\
& +\frac{1-\sum_{i=1}^{m} \beta_{i}}{1-\sum_{i=1}^{m} \alpha_{i}} A-B \\
\leq & -\frac{1-\sum_{i=1}^{m} \beta_{i}}{1-\sum_{i=1}^{m} \alpha_{i}}\left(\sum_{i=1}^{m} \alpha_{i} \sum_{j=0}^{n_{i}-1} \phi^{-1}\left(\sum_{s=0}^{j-1} q(s)\right)\right)-\left(1-\sum_{i=1}^{m} \beta_{i}\right) \sum_{i=0}^{N+1} \phi^{-1}\left(\sum_{s=0}^{i-1} q(s)\right) \\
& -\sum_{i=1}^{m} \beta_{i} \sum_{j=n_{i}}^{N+1} \phi^{-1}\left(\sum_{s=0}^{j-1} q(s)\right)+\frac{1-\sum_{i=1}^{m} \beta_{i}}{1-\sum_{i=1}^{m} \alpha_{i}} A-B<0 .
\end{aligned}
$$


It follows that

$$
\begin{aligned}
\frac{F(c)}{c}= & \frac{1-\sum_{i=1}^{m} \beta_{i}}{1-\sum_{i=1}^{m} \alpha_{i}}\left(\sum_{i=1}^{m} \alpha_{i} \sum_{j=0}^{n_{i}-1} \phi^{-1}\left(1-\frac{\sum_{s=0}^{j-1} f(s, x(s+1), \Delta x(s), \Delta x(s+1))}{\phi(c)}\right)+\frac{A}{c}\right) \\
& +\left(1-\sum_{i=1}^{m} \beta_{i}\right) \sum_{i=0}^{N+1} \phi^{-1}\left(1-\frac{\sum_{s=0}^{i-1} f(s, x(s+1), \Delta x(s), \Delta x(s+1))}{\phi(c)}\right) \\
& +\sum_{i=1}^{m} \beta_{i} \sum_{j=n_{i}}^{N+1} \phi^{-1}\left(1-\frac{\sum_{s=0}^{j-1} f(s, x(s+1), \Delta x(s), \Delta x(s+1))}{\phi(c)}\right)-\frac{B}{c}
\end{aligned}
$$

Note that $f$ is continuous, let Denote

$$
L_{1}=\max \left\{\max _{n \in[0, N+2]}|x(n)|, \max _{n \in[0, N+1]}|\Delta x(n)|: x \in \bar{D}\right\}
$$

and

$$
L_{2}=\max _{j \in[0, N]} f_{L_{1}}(j)=\max _{j \in[0, N]|x|,|y| \leq L_{1},|w| \leq L_{1}}|f(j, x, y, w)| .
$$

Then

$$
\begin{aligned}
& \lim _{c \rightarrow+\infty}\left|\frac{\sum_{s=0}^{i-1} f(s, x(s+1), \Delta x(s), \Delta x(s+1))}{\phi(c)}\right|=0, \quad i \in[0, N+1], \\
& \lim _{c \rightarrow+\infty} \frac{B}{c}=\lim _{c \rightarrow+\infty} \frac{A}{c}=0 .
\end{aligned}
$$

Hence

$\lim _{c \rightarrow+\infty} \frac{F(c)}{c}=\frac{1-\sum_{i=1}^{m} \beta_{i}}{1-\sum_{i=1}^{m} \alpha_{i}} \sum_{i=1}^{m} \alpha_{i} n_{i}+\left(1-\sum_{i=1}^{m} \beta_{i}\right)(N+2)+\sum_{i=1}^{m} \beta_{i}\left(N+2-n_{i}\right)>0$.

Then, together with $F(0)<0$ and $F$ is increasing on $(-\infty,+\infty)$, it follows that there exists unique $A_{x} \in(0,+\infty)$ such that $F\left(A_{x}\right)=0$. Since $F(\phi(\Delta y(0)))=$ 0 , we get that $\phi(\Delta y(0))=A_{x} \in(0,+\infty)$. It follows that $\Delta y(0)>0$.

Step 2. Prove that $\phi(\Delta y(N+1)) \leq 0$. In fact, let

$$
\begin{aligned}
G(c)= & \frac{1-\sum_{i=1}^{m} \beta_{i}}{1-\sum_{i=1}^{m} \alpha_{i}}\left[\sum_{i=0}^{N+1} \phi^{-1}\left(c+\sum_{s=i}^{N} f(s, x(s+1), \Delta x(s), \Delta x(s+1))\right)\right. \\
& \left.-\sum_{i=1}^{m} \alpha_{i} \sum_{j=i}^{N+1} \phi^{-1}\left(c+\sum_{s=j}^{N} f(s, x(s+1), \Delta x(s), \Delta x(s+1))\right)+A\right] \\
& +\sum_{i=1}^{m} \beta_{i} \sum_{j=n_{i}}^{N+1} \phi^{-1}\left(c+\sum_{s=j}^{N} f(s, x(s+1), \Delta x(s), \Delta x(s+1))\right)-B \\
= & \frac{1-\sum_{i=1}^{m} \beta_{i}}{1-\sum_{i=1}^{m} \alpha_{i}}\left[\left(1-\sum_{i=1}^{m} \alpha_{i}\right) \sum_{i=0}^{N+1} \phi^{-1}\left(c+\sum_{s=i}^{N} f(s, x(s+1), \Delta x(s), \Delta x(s+1))\right)\right.
\end{aligned}
$$




$$
\begin{aligned}
& \left.+\sum_{i=1}^{m} \alpha_{i} \sum_{j=0}^{i-1} \phi^{-1}\left(c+\sum_{s=j}^{N} f(s, x(s+1), \Delta x(s), \Delta x(s+1))\right)+A\right] \\
& +\sum_{i=1}^{m} \beta_{i} \sum_{j=n_{i}}^{N+1} \phi^{-1}\left(c+\sum_{s=j}^{N} f(s, x(s+1), \Delta x(s), \Delta x(s+1))\right)-B .
\end{aligned}
$$

Similar to Step 1, we have that

$$
\begin{aligned}
G(0)= & \frac{1-\sum_{i=1}^{m} \beta_{i}}{1-\sum_{i=1}^{m} \alpha_{i}}\left[\left(1-\sum_{i=1}^{m} \alpha_{i}\right) \sum_{i=0}^{N+1} \phi^{-1}\left(\sum_{s=i}^{N} f(s, x(s+1), \Delta x(s), \Delta x(s+1))\right)\right. \\
& \left.+\sum_{i=1}^{m} \alpha_{i} \sum_{j=0}^{i-1} \phi^{-1}\left(\sum_{s=j}^{N} f(s, x(s+1), \Delta x(s), \Delta x(s+1))\right)\right] \\
& +\sum_{i=1}^{m} \beta_{i} \sum_{j=n_{i}}^{N+1} \phi^{-1}\left(\sum_{s=j}^{N} f(s, x(s+1), \Delta x(s), \Delta x(s+1))\right) \\
& +\frac{1-\sum_{i=1}^{m} \beta_{i}}{1-\sum_{i=1}^{m} \alpha_{i}} A-B>0
\end{aligned}
$$

and

$$
\begin{aligned}
\lim _{c \rightarrow-\infty} \frac{G(c)}{c}= & \frac{1-\sum_{i=1}^{m} \beta_{i}}{1-\sum_{i=1}^{m} \alpha_{i}}\left[\left(1-\sum_{i=1}^{m} \alpha_{i}\right)(N+2)+\sum_{i=1}^{m} \alpha_{i} i\right] \\
& +\sum_{i=1}^{m} \beta_{i}\left(N+2-n_{i}\right)>0 .
\end{aligned}
$$

It is easy to see that $G(c)$ is increasing on $(-\infty,+\infty)$. Then there exists unique $D_{x} \leq 0$ such that $G\left(D_{x}\right)=0$. Since $G(\phi(\Delta y(N+1)))=0$, we get that $D_{x}=G(\phi(\Delta y(N+1)))$. Then $\Delta y(N+1) \leq 0$.

It follows from Step 1 and Step 2 and the fact that $\Delta y(n)$ is decreasing on $[0, N+1]$ that there exists unique $n_{0} \in[0, N]$ such that $\Delta y\left(n_{0}\right)>0$ and $\Delta y\left(n_{0}+1\right) \leq 0$.

Lemma 2.3. Suppose that $\left(H_{1}\right),\left(H_{2}\right)$ and $\left(H_{3}\right)$ hold. If $y$ is a solution of $B V P(4)$, then $y(0) \geq 0, y(N+2) \geq 0$ and $y(n)>0$ for all $n \in[1, N+1]$.

Proof. Since $\left(H_{1}\right),\left(H_{2}\right)$ and $\left(H_{3}\right)$ hold, we get from Lemma 2.2 that there exists unique $n_{0} \in[0, N]$ such that $\Delta y\left(n_{0}\right)>0$ and $\Delta y\left(n_{0}+1\right) \leq 0$. It follows from the equation (4) that

$$
\phi(\Delta y(n))=\left\{\begin{array}{l}
\phi\left(\Delta y\left(n_{0}+1\right)\right)-\sum_{i=n_{0}+1}^{n-1} f(k, x(k+1), \Delta x(k), \Delta x(k+1)), n \in\left[n_{0}+1, N+1\right], \\
\phi\left(\Delta y\left(n_{0}\right)\right)+\sum_{i=n}^{n_{0}-1} f(k, x(k+1), \Delta x(k), \Delta x(k+1)), n \in\left[0, n_{0}\right] .
\end{array}\right.
$$


So

$$
y(n)=\left\{\begin{array}{l}
y(N+2)+\sum_{i=n}^{N+1} \phi^{-1}\left(-\phi\left(\Delta y\left(n_{0}+1\right)\right)+\sum_{j=n_{0}+1}^{i-1} f(j, x(j+1), \Delta x(j), \Delta x(j+1))\right), \\
n \in\left[n_{0}+1, N+2\right], \\
y(0)+\sum_{i=0}^{n-1} \phi^{-1}\left(\phi\left(\Delta y\left(n_{0}\right)\right)+\sum_{j=i}^{n_{0}-1} f(j, x(j+1), \Delta x(j), \Delta x(j+1))\right), \\
n \in\left[0, n_{0}+1\right]
\end{array}\right.
$$

with

$$
\begin{aligned}
& y\left(n_{0}+1\right) \\
= & y(0)+\sum_{0}^{n_{0}} \phi^{-1}\left(\phi\left(\Delta y\left(n_{0}\right)\right)+\sum_{j=i}^{n_{0}-1} f(j, x(j+1), \Delta x(j), \Delta x(j+1))\right) \\
= & y(N+2)+\sum_{i=n_{0}+1}^{N+1} \phi^{-1}\left(-\phi\left(\Delta y\left(n_{0}+1\right)\right)+\sum_{j=n_{0}+1}^{i-1} f(j, x(j+1), \Delta x(j), \Delta x(j+1))\right) .
\end{aligned}
$$

It follows from $\left(H_{1}\right),\left(H_{2}\right)$ and Lemma 2.2 that

$$
y(n) \geq\left\{\begin{array}{l}
y(N+2), n \in\left[n_{0}+1, N+2\right], \\
y(0), n \in\left[0, n_{0}+1\right] .
\end{array}\right.
$$

So $y(n)>\min \{y(0), y(N+2)\}$ for all $n \in[0, N+2]$. From BCs in BVP(4), we see that

$$
\begin{aligned}
y(0) & \geq \sum_{i=1}^{m} \alpha_{i}\{y(0), y(N+2)\}+A \geq \sum_{i=1}^{m} \alpha_{i}\{y(0), y(N+2)\}, \\
y(N+2) & \geq \sum_{i=1}^{m} \beta_{i} \min \{y(0), y(N+2)\}+B \geq \sum_{i=1}^{m} \beta_{i} \min \{y(0), y(N+2)\} .
\end{aligned}
$$

Then

$$
\min \{y(0), y(N+2)\} \geq \min \left\{\sum_{i=1}^{m} \alpha_{i}, \sum_{i=1}^{m} \beta_{i}\right\} \min \{y(0), y(N+2)\},
$$

that is,

$$
\left(1-\min \left\{\sum_{i=1}^{m} \alpha_{i}, \sum_{i=1}^{m} \beta_{i}\right\}\right) \min \{y(0), y(N+2)\} \geq 0 .
$$

It follow from $\left(H_{2}\right)$ that $\min \{y(0), y(N+2)\} \geq 0$. Hence $y(n)>0$ for all $n \in[1, N+1]$. 
Lemma 2.4. Suppose that $\left(H_{1}\right),\left(H_{2}\right)$ and $\left(H_{3}\right)$ hold. If $y$ is a solution of $B V P(4)$, then

(7)

$$
x(n) \geq \sigma \max _{n=0}^{N+2}|x(n)| \text { for all } n \in[1, N+1], \sigma=\min \left\{\frac{n}{N+2}, \frac{N+2-n}{N+2}\right\} .
$$

Proof. It follow from Lemma 2.2 and Lemma 2.3 that $y(n) \geq 0$ for $n \in[0, N+2]$. Suppose that $y\left(n_{0}\right)=\max \{y(n): n \in[0, N+2]\}$. Since $\Delta y(0)>0$ and $\Delta y(N+1) \leq 0$, we get that $n_{0} \in[1, N+1]$. For $n \in\left[1, n_{0}\right]$, it is easy to see that

$$
\begin{aligned}
& \frac{y\left(n_{0}\right)-x(0)}{n_{0}} n+y(0)-y(n) \\
= & \frac{n \sum_{s=0}^{n_{0}-1} \Delta y(s)-n_{0} \sum_{s=0}^{n-1} \Delta y(s)}{n_{0}} \\
= & \frac{-\left(n_{0}-n\right) \sum_{s=0}^{n-1} \Delta y(s)+n \sum_{s=n}^{n_{0}-1} \Delta y(s)}{n_{0}} .
\end{aligned}
$$

Since $\Delta[\phi(\Delta y(n)]=-f(n, x(n), x(n+1), \Delta x(n), \Delta x(n+1))<0$ for all $n \in$ $[0, N]$, we get that $\Delta y(s) \leq \Delta y(j)$ for all $s \geq j$. Then $\left(n_{0}-n\right) \sum_{s=0}^{n-1} \Delta y(s) \leq$ $n \sum_{s=n}^{n_{0}-1} \Delta y(s)$. It follows that $\frac{y\left(n_{0}\right)-y(0)}{n_{0}} n+y(0)-y(n) \leq 0$. Then $y(n) \geq \frac{n}{n_{0}} y\left(n_{0}\right)+\left(1-\frac{n}{n_{0}}\right) y(0) \geq \frac{n}{N+2} \max _{n \in[0, N+2]} y(n)$ for all $n \in\left[1, n_{0}\right]$.

Similarly, if $n \in\left[n_{0}, N+1\right]$, we get

$$
y(n) \geq \frac{N+2-n}{N+2} \max _{n \in[0, N+2]} y(n) \text { for all } n \in\left[n_{0}, N+1\right] .
$$

Then

$$
y(n) \geq \min \left\{\frac{n}{N+2}, \frac{N+2-n}{N+2}\right\} \max _{n=0}^{N+2}|y(n)| \text { for all } n \in[0, N+2] .
$$

Lemma 2.5. Suppose that $\left(H_{1}\right),\left(H_{2}\right)$ and $\left(H_{3}\right)$ hold. If $y$ is a solution of $B V P(4)$, then

(8) $y(n)=B_{x}+\sum_{i=0}^{n-1} \phi^{-1}\left(A_{x}-\sum_{j=0}^{i-1} f(j, x(j+1), \Delta x(j), \Delta x(j+1))\right)$,

where $A_{x}$ satisfies

$$
\begin{aligned}
& \frac{1-\sum_{i=1}^{m} \beta_{i}}{1-\sum_{i=1}^{m} \alpha_{i}}\left(\sum_{i=1}^{m} \alpha_{i} \sum_{j=0}^{n_{i}-1} \phi^{-1}\left(A_{x}-\sum_{s=0}^{j-1} f(s, x(s+1), \Delta x(s), \Delta x(s+1))\right)+A\right) \\
& +\sum_{i=0}^{N+1} \phi^{-1}\left(A_{x}-\sum_{s=0}^{i-1} f(s, x(s+1), \Delta x(s), \Delta x(s+1))\right)
\end{aligned}
$$


$-\sum_{i=1}^{m} \beta_{i} \sum_{j=0}^{n_{i}-1} \phi^{-1}\left(A_{x}-\sum_{s=0}^{j-1} f(s, x(s+1), \Delta x(s), \Delta x(s+1))\right)-B=0$

and $B_{x}$ satisfies

$$
B_{x}=\frac{\sum_{i=1}^{m} \alpha_{i} \sum_{j=0}^{n_{i}-1} \phi^{-1}\left(A_{x}-\sum_{s=0}^{j-1} f(s, x(s+1), \Delta x(s), \Delta x(s+1))\right)+A}{1-\sum_{i=1}^{m} \alpha_{i}} .
$$

Proof. Since $y$ is a solution of $\operatorname{BVP}(4)$, we get

$$
\phi(\Delta y(n))=\phi(\Delta x(0))-\sum_{k=0}^{n-1} f(k, x(k+1), \Delta x(k), \Delta x(k+1)) .
$$

Then

$$
y(n)=y(0)+\sum_{i=0}^{n-1} \phi^{-1}\left(\phi(\Delta x(0))-\sum_{j=0}^{i-1} f(j, x(j+1), \Delta x(j), \Delta x(j+1))\right) .
$$

It follows from the BCs in (4) that

$$
y(0)=\sum_{i=1}^{m} \alpha_{i}\left(y(0)+\sum_{i=0}^{n_{i}-1} \phi^{-1}\left(\phi(\Delta y(0))-\sum_{j=0}^{i-1} f(j, x(j+1), \Delta x(j), \Delta x(j+1))\right)\right)+A
$$

and

$$
\begin{aligned}
& y(0)+\sum_{i=0}^{N+1} \phi^{-1}\left(\phi(\Delta y(0))-\sum_{j=0}^{i-1} f(j, x(j+1), \Delta x(j), \Delta x(j+1))\right) \\
= & \sum_{i=1}^{m} \beta_{i}\left(y(0)+\sum_{i=0}^{n_{i}-1} \phi^{-1}\left(\phi(\Delta y(0))-\sum_{j=0}^{i-1} f(j, x(j+1), \Delta x(j), \Delta x(j+1))\right)\right)+B .
\end{aligned}
$$

Then

$$
\begin{aligned}
& \frac{1-\sum_{i=1}^{m} \beta_{i}}{1-\sum_{i=1}^{m} \alpha_{i}}\left[\sum_{i=1}^{m} \alpha_{i} \sum_{j=0}^{n_{i}-1} \phi^{-1}\left(\phi(\Delta y(0))-\sum_{s=0}^{j-1} f(s, x(s+1), \Delta x(s), \Delta x(s+1))\right)+A\right] \\
= & \sum_{i=1}^{m} \beta_{i} \sum_{j=0}^{n_{i}-1} \phi^{-1}\left(\phi(\Delta y(0))-\sum_{s=0}^{j-1} f(s, x(s+1), \Delta x(s), \Delta x(s+1))\right)+B \\
& -\sum_{i=0}^{N+1} \phi^{-1}\left(\phi(\Delta y(0))-\sum_{j=0}^{i-1} f(j, x(j+1), \Delta x(j), \Delta x(j+1))\right) .
\end{aligned}
$$


It follows from Step 1 in the proof of Lemma 2.2 that $\phi(\Delta y(0))=A_{x}$ and $A_{x}>0$ satisfies

$$
\begin{aligned}
& \frac{1-\sum_{i=1}^{m} \beta_{i}}{1-\sum_{i=1}^{m} \alpha_{i}}\left(\sum_{i=1}^{m} \alpha_{i} \sum_{j=0}^{n_{i}-1} \phi^{-1}\left(A_{x}-\sum_{s=0}^{j-1} f(s, x(s+1), \Delta x(s), \Delta x(s+1))\right)+A\right) \\
& +\sum_{i=0}^{N+1} \phi^{-1}\left(A_{x}-\sum_{s=0}^{i-1} f(s, x(s+1), \Delta x(s), \Delta x(s+1))\right) \\
& -\sum_{i=1}^{m} \beta_{i} \sum_{j=0}^{n_{i}-1} \phi^{-1}\left(A_{x}-\sum_{s=0}^{j-1} f(s, x(s+1), \Delta x(s), \Delta x(s+1))\right)-B=0
\end{aligned}
$$

and $B_{x}$ satisfies (9).

Lemma 2.6. Suppose that $\left(H_{1}\right),\left(H_{2}\right)$ and $\left(H_{3}\right)$ hold. If $y$ is a solution of $B V P(4)$, then there exists a $n_{0} \in[0, N]$ such that

$$
\begin{aligned}
\max _{n \in[0, N+2]} y(n)= & y\left(n_{0}+1\right) \\
\geq \max & \left\{\sum_{i=0}^{n_{0}} \phi^{-1}\left(\sum_{j=i}^{n_{0}-1} f(j, x(j+1), \Delta x(j), \Delta x(j+1))\right),\right. \\
& \left.\sum_{i=n_{0}+1}^{N+1} \phi^{-1}\left(\sum_{j=n_{0}+1}^{i-1} f(j, x(j+1), \Delta x(j), \Delta x(j+1))\right)\right\} .
\end{aligned}
$$

Proof. It follows from Lemma 2.2 that there is $n_{0} \in[0, N]$ such that $\Delta y\left(n_{0}\right)>0$ and $\Delta y\left(n_{0}+1\right) \leq 0, \Delta y(n)>0$ for all $n \in\left[0, n_{0}\right]$ and $\Delta y(n) \leq 0$ for all $n \in\left[n_{0}+1, N+1\right]$. Then

$$
\max _{n \in[0, N+2]} y(n)=y\left(n_{0}+1\right)
$$

and there exists $\xi \in\left(n_{0}, n_{0}+1\right]$ such that

$$
\frac{\Delta y\left(n_{0}+1\right)-\Delta y\left(n_{0}\right)}{n_{0}+1-n_{0}}=\frac{0-\Delta y\left(n_{0}\right)}{\xi-n_{0}} .
$$

Then

$$
\Delta x\left(n_{0}+1\right)=-\frac{n_{0}+1-\xi}{\xi-n_{0}} \Delta x\left(n_{0}\right) .
$$

It is easy to see from (4) that

$$
\begin{array}{r}
0<\Delta y\left(n_{0}\right)=\phi^{-1}\left(A_{x}-\sum_{i=0}^{n_{0}-1} f(i, x(i+1), \Delta x(i), \Delta x(i+1))\right), \\
0 \geq \Delta y\left(n_{0}+1\right)=\phi^{-1}\left(A_{x}-\sum_{i=0}^{n_{0}} f(i, x(i+1), \Delta x(i), \Delta x(i+1))\right),
\end{array}
$$


where $A_{x}=\phi(\Delta y(0))$. So (10) implies that

$$
\begin{aligned}
& \phi^{-1}\left(A_{x}-\sum_{i=0}^{n_{0}} f(i, x(i+1), \Delta x(i), \Delta x(i+1))\right) \\
= & -\frac{n_{0}+1-\xi}{\xi-n_{0}} \phi^{-1}\left(A_{x}-\sum_{i=0}^{n_{0}-1} f(i, x(i+1), \Delta x(i), \Delta x(i+1))\right) .
\end{aligned}
$$

Then

$$
\begin{aligned}
A_{x}= & \frac{1}{1+\phi\left(\frac{n_{0}+1-\xi}{\xi-n_{0}}\right)}\left(\sum_{i=0}^{n_{0}} f(i, x(i+1), \Delta x(i), \Delta x(i+1))\right. \\
& \left.+\phi\left(\frac{n_{0}+1-\xi}{\xi-n_{0}}\right) \sum_{i=0}^{n_{0}-1} f(i, x(i+1), \Delta x(i), \Delta x(i+1))\right) .
\end{aligned}
$$

We get that

$$
\begin{aligned}
& \sum_{i=0}^{n_{0}-1} f(i, x(i+1), \Delta x(i), \Delta x(i+1)) \\
\leq & A_{x} \leq \sum_{i=0}^{n_{0}} f(i, x(i+1), \Delta x(i), \Delta x(i+1)) .
\end{aligned}
$$

Lemma 2.3 implies that $B_{x}=y(0) \geq 0$. Furthermore, one has from (11) that

$$
\begin{aligned}
& y\left(n_{0}+1\right) \\
= & B_{x}+\sum_{i=0}^{n_{0}} \phi^{-1}\left(A_{x}-\sum_{j=0}^{i-1} f(j, x(j+1), \Delta x(j), \Delta x(j+1))\right) \\
\geq & \sum_{i=0}^{n_{0}} \phi^{-1}\left(\sum_{j=0}^{n_{0}-1} f(j, x(j+1), \Delta x(j), \Delta x(j+1))-\sum_{j=0}^{i-1} f(j, x(j+1), \Delta x(j), \Delta x(j+1))\right) \\
= & \sum_{i=0}^{n_{0}} \phi^{-1}\left(\sum_{j=i}^{n_{0}-1} f(j, x(j+1), \Delta x(j), \Delta x(j+1))\right) .
\end{aligned}
$$

On the other hand, we have similarly to Lemma 2.2 and Lemma 2.3 that there exist constants $\overline{A_{x}}=\phi(\Delta y(N+1))$ and $\overline{B_{x}}=y(N+2)$ such that

$$
\Delta y(n)=\phi^{-1}\left(\overline{A_{x}}+\sum_{j=n}^{N} f(j, x(j+1), \Delta x(j), \Delta x(j+1))\right)
$$

and

$$
y(n)=\overline{B_{x}}-\sum_{i=n}^{N+1} \phi^{-1}\left(\overline{A_{x}}+\sum_{j=i}^{N} f(j, x(j+1), \Delta x(j), \Delta x(j+1))\right) .
$$


It follows that

$$
\begin{gathered}
\Delta y\left(n_{0}\right)=\phi^{-1}\left(\overline{A_{x}}+\sum_{i=n_{0}}^{N} f(i, x(i+1), \Delta x(i), \Delta x(i+1))\right)>0, \\
\Delta x\left(n_{0}+1\right)=\phi^{-1}\left(\overline{A_{x}}+\sum_{i=n_{0}+1}^{N} f(i, x(i+1), \Delta x(i), \Delta x(i+1))\right) \leq 0 .
\end{gathered}
$$

So

$$
\begin{aligned}
& \phi^{-1}\left(\overline{A_{x}}+\sum_{i=n_{0}+1}^{N} f(i, x(i+1), \Delta x(i), \Delta x(i+1))\right) \\
= & -\frac{n_{0}+1-\xi}{\xi-n_{0}} \phi^{-1}\left(\overline{A_{x}}+\sum_{i=n_{0}}^{N} f(i, x(i+1), \Delta x(i), \Delta x(i+1))\right) .
\end{aligned}
$$

Then

$$
\begin{aligned}
\overline{A_{x}}= & -\frac{1}{1+\phi\left(\frac{n_{0}+1-\xi}{\xi-n_{0}}\right)}\left(\sum_{i=n_{0}+1}^{N} f(i, x(i+1), \Delta x(i), \Delta x(i+1))\right. \\
& \left.+\phi\left(\frac{n_{0}+1-\xi}{\xi-n_{0}}\right) \sum_{i=n_{0}}^{N} f(i, x(i+1), \Delta x(i), \Delta x(i+1))\right) .
\end{aligned}
$$

We get that

$$
\begin{gathered}
-\sum_{i=n_{0}+1}^{N} f(i, x(i+1), \Delta x(i), \Delta x(i+1)) \\
\geq \overline{A_{x}} \geq-\sum_{i=n_{0}}^{N} f(i, x(i+1), \Delta x(i), \Delta x(i+1)) .
\end{gathered}
$$

One sees from Lemma 2.3 that $\overline{B_{x}}=x(N+2) \geq 0$. Hence

$$
\begin{aligned}
y\left(n_{0}+1\right)= & \overline{B_{x}}-\sum_{i=n_{0}+1}^{N+1} \phi^{-1}\left(\overline{A_{x}}+\sum_{j=i}^{N} f(j, x(j+1), \Delta x(j), \Delta x(j+1))\right) \\
\geq & \sum_{i=n_{0}+1}^{N+1} \phi^{-1}\left(\sum_{j=n_{0}+1}^{N} f(j, x(j+1), \Delta x(j), \Delta x(j+1))\right. \\
& \left.-\sum_{j=i}^{N} f(j, x(j+1), \Delta x(j), \Delta x(j+1))\right) \\
= & \sum_{i=n_{0}+1}^{N+1} \phi^{-1}\left(\sum_{j=n_{0}+1}^{i-1} f(j, x(j+1), \Delta x(j), \Delta x(j+1))\right) .
\end{aligned}
$$


Hence

$$
\begin{aligned}
\max _{n \in[0, N+2]} y(n)= & y\left(n_{0}+1\right) \\
\geq & \max \left\{\sum_{i=0}^{n_{0}} \phi^{-1}\left(\sum_{j=i}^{n_{0}-1} f(j, x(j+1), \Delta x(j), \Delta x(j+1))\right),\right. \\
& \left.\sum_{i=n_{0}+1}^{N+1} \phi^{-1}\left(\sum_{j=n_{0}+1}^{i-1} f(j, x(j+1), \Delta x(j), \Delta x(j+1))\right)\right\} .
\end{aligned}
$$

Define the operator $T: P \rightarrow X$ by

$(T x)(n)=B_{x}+\sum_{i=0}^{n-1} \phi^{-1}\left(A_{x}-\sum_{j=0}^{i-1} f(j, x(j), x(j+1), \Delta x(j))\right), n \in[0, N+2]$

for $x \in P$, where $A_{x}$ satisfies

$$
\begin{aligned}
& \frac{1-\sum_{i=1}^{m} \beta_{i}}{1-\sum_{i=1}^{m} \alpha_{i}}\left(\sum_{i=1}^{m} \alpha_{i} \sum_{j=0}^{n_{i}-1} \phi^{-1}\left(A_{x}-\sum_{s=0}^{j-1} f(s, x(s+1), \Delta x(s), \Delta x(s+1))\right)+A\right) \\
& +\sum_{i=0}^{N+1} \phi^{-1}\left(A_{x}-\sum_{s=0}^{i-1} f(s, x(s+1), \Delta x(s), \Delta x(s+1))\right) \\
& -\sum_{i=1}^{m} \beta_{i} \sum_{j=0}^{n_{i}-1} \phi^{-1}\left(A_{x}-\sum_{s=0}^{j-1} f(s, x(s+1), \Delta x(s), \Delta x(s+1))\right)-B=0
\end{aligned}
$$

and $B_{x}$ satisfies

$$
B_{x}=\frac{1}{1-\sum_{i=1}^{m} \alpha_{i}}\left(\sum_{i=1}^{m} \alpha_{i} \sum_{j=0}^{n_{i}-1} \phi^{-1}\left(A_{x}-\sum_{s=0}^{j-1} f(s, x(s+1), \Delta x(s), \Delta x(s+1))\right)+A\right) .
$$

Lemma 2.7. Suppose that $\left(H_{1}\right)$ and $\left(H_{2}\right)$ hold. Then

(i) Tx satisfies the followings:

$$
\left\{\begin{array}{l}
\Delta[\phi(\Delta(T x)(n))]+f(n, x(n+1), \Delta x(n+1), \Delta x(n+1))=0 \\
(T x)(0)-\sum_{i=1}^{m} \alpha_{i}(T x)\left(n_{i}\right)=A \\
(T x)(N+2)-\sum_{i=1}^{m} \beta_{i}(T x)\left(n_{i}\right)=B
\end{array}\right.
$$

(ii) $T x \in P$ for each $x \in P$;

(iii) $x$ is a solution of $B V P(4)$ if and only if $x$ is a solution of the operator equation $x=T x$;

(iv) $T: P \rightarrow P$ is completely continuous.

Proof. (i) By the definition of Tx, we get (12).

(ii) Note the definition of $P$. Since $\left(H_{1}\right),\left(H_{2}\right)$ and $\left(H_{3}\right)$ hold for $x \in P,(12)$, Lemma 2.2, Lemma 2.3 and Lemma 2.4 imply that $\Delta(T x)(n)$ is decreasing on 
$[0, N+1]$ and $(T x)(n) \geq \sigma \max _{n \in[0, N+2]}(T x)(n)$ for all $n \in[0, N+2]$. It follows that $T x \in P$.

(iii) It is easy to see from (12) that $x$ is a solution of $\operatorname{BVP}(4)$ if and only if $x$ is a solution of the operator equation $x=T x$.

(iv) It suffices to prove that $T$ is continuous on $P$ and $T$ is relative compact. We divide the proof into three steps:

Step 1. For each bounded subset $D \subset P$, prove that $\left\{\left(A_{x}, B_{x}\right): x \in \bar{D}\right\}$ is bounded in $\mathbb{R}^{2}$.

Denote

$$
L_{1}=\max \left\{\max _{n \in[0, N+2]}|x(n)|, \max _{n \in[0, N+1]}|\Delta x(n)|: x \in \bar{D}\right\}
$$

and

$$
L_{2}=\max _{j \in[0, N]} f_{L_{1}}(j)=\max _{j \in[0, N]|x|,|y| \leq L_{1},|w| \leq L_{1}}|f(j, x, y, w)| .
$$

It follows from (11) in the proof of Lemma 2.6 that

$$
0 \leq A_{x} \leq \sum_{j=0}^{N} f(j, x(j+1), \Delta x(j), \Delta x(j+1)) \leq(N+1) L_{2} .
$$

Then

$$
\begin{aligned}
\left|B_{x}\right|= & \left|\frac{1}{1-\sum_{i=1}^{m} \alpha_{i}} \sum_{j=1}^{m} \alpha_{j} \sum_{i=0}^{n_{j}-1} \phi^{-1}\left(A_{x}-\sum_{s=0}^{i-1} f(s, x(s+1), \Delta x(s), \Delta x(s+1))\right)\right| \\
& +\frac{A}{1-\sum_{i=1}^{m} \alpha_{i}} \\
\leq & \frac{1}{1-\sum_{i=1}^{m} \alpha_{i}} \sum_{j=1}^{m} \alpha_{j} \sum_{i=0}^{n_{j}-1} \phi^{-1}\left((N+1) L_{2}+i L_{2}\right)+\frac{A}{1-\sum_{i=1}^{m} \alpha_{i}} .
\end{aligned}
$$

Hence $\left\{\left(A_{x}, B_{x}\right): x \in \bar{D}\right\}$ is bounded in $\mathbb{R}^{2}$.

Step 2. For each bounded subset $D \subset P$, and each $x_{0} \in D$, prove that $T$ is continuous at $x_{0}$.

For $x_{0} \in D$ and $x_{n} \in D$ with $x_{n} \rightarrow x_{0}(n \rightarrow+\infty)$ in $D$. Denote $u_{n}(k)=$ $\left(T x_{n}\right)(k), u_{0}(k)=\left(T x_{0}\right)(k)$ for all $k \in[0, N+2]$. We prove that $T$ is continuous at $x_{0}$, i.e., $u_{n} \rightarrow u_{0}(n \rightarrow+\infty)$. Let $A_{x_{0}}, B_{x_{0}}$ be defined by

$$
\begin{aligned}
& \frac{1-\sum_{i=1}^{m} \beta_{i}}{1-\sum_{i=1}^{m} \alpha_{i}}\left(\sum_{i=1}^{m} \alpha_{i} \sum_{j=0}^{n_{i}-1} \phi^{-1}\left(A_{x_{0}}-\sum_{s=0}^{j-1} f(s, x(s+1), \Delta x(s), \Delta x(s+1))\right)+A\right) \\
& +\sum_{i=0}^{N+1} \phi^{-1}\left(A_{x_{0}}-\sum_{s=0}^{i-1} f(s, x(s+1), \Delta x(s), \Delta x(s+1))\right)
\end{aligned}
$$




$$
-\sum_{i=1}^{m} \beta_{i} \sum_{j=0}^{n_{i}-1} \phi^{-1}\left(A_{x_{0}}-\sum_{s=0}^{j-1} f(s, x(s+1), \Delta x(s), \Delta x(s+1))\right)-B=0
$$

and $B_{x}$ satisfies

$$
B_{x_{0}}=\frac{1}{1-\sum_{i=1}^{m} \alpha_{i}}\left(\sum_{i=1}^{m} \alpha_{i} \sum_{j=0}^{n_{i}-1} \phi^{-1}\left(A_{x_{0}}-\sum_{s=0}^{j-1} f(s, x(s+1), \Delta x(s), \Delta x(s+1))\right)+A\right) .
$$

First, we prove that $A_{x}, B_{x}$ are continuous in $x$, i.e.,

$$
\left(A_{x_{n}}, B_{x_{n}}\right) \rightarrow\left(A_{x_{0}}, B_{x_{0}}\right), \quad n \rightarrow+\infty .
$$

It follows from Step 1 that $\left(A_{x_{n}}, B_{x_{n}}\right)$ is bounded. Without loss of generality, suppose that $\left(A_{x_{n}}, B_{x_{n}}\right) \rightarrow(\bar{A}, \bar{B}) \neq\left(A_{x_{0}}, B_{x_{0}}\right)$.

It is easy to see that

$$
\begin{aligned}
& \lim _{n \rightarrow+\infty} u_{n}(k) \\
= & \lim _{n \rightarrow+\infty}\left[B_{x_{n}}+\sum_{i=0}^{k-1} \phi^{-1}\left(A_{x_{n}}-\sum_{j=0}^{i-1} f\left(j, x_{n}(j+1), \Delta x_{n}(j), \Delta x_{n}(j+1)\right)\right)\right] \\
= & \bar{B}+\sum_{i=0}^{k-1} \phi^{-1}\left(\bar{A}-\lim _{n \rightarrow+\infty} \sum_{j=0}^{i-1} f\left(j, x_{n}(j+1), \Delta x_{n}(j), \Delta x_{n}(j+1)\right)\right) \\
= & \bar{B}+\sum_{i=0}^{k-1} \phi^{-1}\left(\bar{A}-\lim _{n \rightarrow+\infty} \sum_{j=0}^{i-1} f\left(j, x_{0}(j+1), \Delta x_{0}(j), \Delta x_{0}(j+1)\right)\right) \\
= & \bar{u}(k) .
\end{aligned}
$$

It is easy to see that $\bar{u}$ satisfies

$$
\bar{u}(0)-\sum_{i=1}^{m} \alpha_{i} \bar{u}\left(n_{i}\right)=A, \quad \bar{u}(N+2)-\sum_{i=1}^{m} \beta_{i} \bar{u}\left(n_{i}\right)=B .
$$

So

$$
\begin{aligned}
& \frac{1-\sum_{i=1}^{m} \beta_{i}}{1-\sum_{i=1}^{m} \alpha_{i}}\left(\sum_{i=1}^{m} \alpha_{i} \sum_{j=0}^{n_{i}-1} \phi^{-1}\left(\bar{A}-\sum_{s=0}^{j-1} f(s, x(s+1), \Delta x(s), \Delta x(s+1))\right)+A\right) \\
& +\sum_{i=0}^{N+1} \phi^{-1}\left(\bar{A}-\sum_{s=0}^{i-1} f(s, x(s+1), \Delta x(s), \Delta x(s+1))\right) \\
& -\sum_{i=1}^{m} \beta_{i} \sum_{j=0}^{n_{i}-1} \phi^{-1}\left(\bar{A}-\sum_{s=0}^{j-1} f(s, x(s+1), \Delta x(s), \Delta x(s+1))\right)-B=0
\end{aligned}
$$


and $B_{x}$ satisfies

$$
\bar{B}=\frac{1}{1-\sum_{i=1}^{m} \alpha_{i}}\left(\sum_{i=1}^{m} \alpha_{i} \sum_{j=0}^{n_{i}-1} \phi^{-1}\left(\bar{A}-\sum_{s=0}^{j-1} f(s, x(s+1), \Delta x(s), \Delta x(s+1))\right)+A\right) .
$$

It follows from the proof of Step 1 in Lemma 2.2 that $\bar{A}=A_{x_{0}}$, then $\bar{B}=B_{x_{0}}$. Hence

$$
\left(A_{x_{n}}, B_{x_{n}}\right) \rightarrow(\bar{A}, \bar{B})=\left(A_{x_{0}}, B_{x_{0}}\right), \quad n \rightarrow+\infty .
$$

It follows that

$$
\lim _{n \rightarrow+\infty} T x_{n}=T x_{0} .
$$

This implies that $T$ is continuous at $x_{0}$.

Step 3. For each bounded subset $D \subset P$, prove that $T$ is relative compact on $D$.

In fact, for each bounded subset $\Omega \subseteq D$, and $x \in \Omega$. Suppose

$$
\|x\|=\max \left\{\max _{n \in[0, N+2]}|x(n)|, \max _{n \in[0, N+1]}|\Delta x(n)|\right\}<M_{1}
$$

and Step 1 implies that there exist constants $M_{2}>0$ such that $\left|A_{x}\right|,\left|B_{x}\right|<M_{2}$. Then

$$
\begin{aligned}
|(T x)(n)| & =\left|B_{x}+\sum_{i=0}^{n-1} \phi^{-1}\left(A_{x}-\sum_{j=0}^{i-1} f(j, x(j+1), \Delta x(j), \Delta x(j+1))\right)\right| \\
& \leq M_{2}+\sum_{i=0}^{N+1} \phi^{-1}\left(M_{2}+\sum_{j=i}^{N}|f(j, x(j+1), \Delta x(j), \Delta x(j+1))|\right) \\
& \leq M_{2}+\sum_{i=0}^{N+1} \phi^{-1}\left(M_{2}+\sum_{j=i}^{N}|f(j, x(j+1), \Delta x(j), \Delta x(j+1))|\right) \\
& \leq M_{2}+\sum_{i=0}^{N+1} \phi^{-1}\left(M_{2}+\sum_{j=i}^{N} f_{M_{1}}(j)\right) \\
& =: M_{3},
\end{aligned}
$$

where $f_{M_{1}}(j)=\max _{|x| \leq M_{1},|y| \leq M_{1},|w| \leq M_{1}}|f(j, x, y, w)|$. Similarly, one has that

$$
\begin{aligned}
|\Delta(T x)(n)| & =\left|\phi^{-1}\left(A_{x}+\sum_{j=0}^{n-1} f(j, x(j+1), \Delta x(j), \Delta x(j+1))\right)\right| \\
& \leq \phi^{-1}\left(M_{2}+\sum_{j=0}^{n-1}|f(j, x(j+1), \Delta x(j), \Delta x(j+1))|\right)
\end{aligned}
$$




$$
\leq \phi^{-1}\left(M_{2}+\sum_{j=0}^{N} f_{M_{1}}(j)\right)=: M_{4} .
$$

It follows that $T \Omega$ is bounded. Since $X=\mathbb{R}^{N+3}$, one knows that $T \Omega$ is relative compact. Steps 1, 2 and 3 imply that $T$ is completely continuous.

For a group of constants $r_{1}, r_{2}, L_{1}, L_{2}$ and $b$, denote

$$
\begin{aligned}
Q & =\frac{1}{1-\sum_{i=1}^{m} \alpha_{i}} \sum_{j=1}^{m} \alpha_{j} \sum_{i=0}^{n_{j}-1} \phi^{-1}(N-i+1)+\sum_{i=0}^{N+1} \phi^{-1}(N-i+1), \\
M_{2} & =\phi\left(\frac{r_{2}-\frac{A}{1-\sum_{i=1}^{m} \alpha_{i}}}{Q}\right), \quad N_{2}=\frac{\phi\left(L_{2}\right)}{2 N+2}, \\
M_{1} & =\phi\left(\frac{r_{1}-\frac{A}{1-\sum_{i=1}^{m} \alpha_{i}}}{Q}\right), \quad N_{1}=\frac{\phi\left(L_{1}\right)}{2 N+2}, \\
O & =\sigma_{0} \max \left\{\sum_{i=[(N+2) / 2]+1}^{N+2-k} \phi^{-1}(i-[(N+2) / 2]-1), \sum_{i=k}^{[(N+2) / 2]} \phi^{-1}([(N+2) / 2]-i+)\right\}, \\
M_{3} & =\phi\left(\frac{b}{O}\right) .
\end{aligned}
$$

Theorem L. Suppose that $\left(H_{1}\right),\left(H_{2}\right),\left(H_{3}\right)$ hold. Choose $k$ satisfying $0<k<$ $\left[\frac{N+2}{2}\right]$ and denote $\sigma_{0}=\min \left\{\frac{k}{N+2}, \frac{N+2-k}{N+2}\right\}$. Furthermore, there exist positive constants $r_{1}, r_{2}, L_{1}, L_{2}$ and $b$ such that

$$
r_{2}>d=\frac{b}{\sigma_{0}}>b>r_{1}>\frac{A}{1-\sum_{i=1}^{m} \alpha_{i}}, \quad L_{2}>L_{1} .
$$

Let $M_{1}, M_{2}, N_{1}, N_{2}, M_{3}$ be defined above. If $M_{3}<\min \left\{M_{2}, N_{2}\right\}$ and

$\left(A_{1}\right) f(n, u, v, w)<\min \left\{M_{1}, N_{1}\right\}$ for all $n \in[0, N], u \in\left[0, r_{1}\right], w, v \in$ $\left[-L_{1}, L_{1}\right]$

$\left(A_{2}\right) f(n, u, v, w)>M_{3}$ for all $n \in[k, N+2-k], u \in\left[b, b / \sigma_{0}\right], w, v \in$ $\left[-L_{2}, L_{2}\right]$

$\left(A_{3}\right) f(n, u, v, w) \leq \min \left\{M_{2}, N_{2}\right\}$ for all $n \in[0, N], u \in\left[0, r_{2}\right], w, v \in$ $\left[-L_{2}, L_{2}\right]$

then $B V P(3)$ has at least three positive solutions $x_{1}, x_{2}, x_{3}$ such that

(i) $x_{1}$ satisfies $\max _{n \in[0, N+2]} x_{1}(n)<r_{1}$ and $\max _{n \in[0, N+1]}\left|\Delta x_{1}(n)\right|<L_{1}$;

(ii) $x_{2}$ satisfies $b \leq \min _{t \in[k, N+2-k]} x_{2}(n)<\max _{n \in[0, N+2]} x_{2}(n) \leq r_{2}$ and $\max _{n \in[0, N+1]}\left|\Delta x_{2}(n)\right| \leq L_{2}$;

(iii) $x_{3}$ satisfies $\max _{n \in[0, N+2]} x(n) \leq r_{2}, \max _{n \in[0, N+1]}|\Delta x(n)| \leq L_{2}$, $\min _{t \in[k, N+2-k]} x_{3}(n)<b, \max _{n \in[0, N+2]} x_{3}(n)>r_{1}$, and $\max _{n \in[0, N+2]}\left|\Delta x_{3}(n)\right|$ $>L_{1}$.

Proof. To apply Lemma 2.1, we prove that all conditions in Lemma 2.1 are satisfied. By the definitions, it is easy to see that $\alpha$ and $\beta$ are two nonnegative 
continuous convex functionals on the cone $P$ satisfying $(a)$ and $(b), \psi$ is a nonnegative continuous concave functional on the cone $P$ with $\psi(x) \leq \alpha(x)$ for all $x \in P$. Lemma 2.7 implies that $x=x(n)$ is a solution of $\operatorname{BVP}(3)$ if and only if $x$ is a solution of the operator equation $x=T x$ and $T: P \rightarrow P$ is completely continuous.

Now, we prove all conditions of Lemma 2.1 hold.

Step 1. Prove that $T \bar{P}\left(\alpha, r_{2} ; \beta, L_{2}\right) \subseteq \bar{P}\left(\alpha, r_{2} ; \beta, L_{2}\right)$.

For $x \in \bar{P}\left(\alpha, r_{2} ; \beta, L_{2}\right)$, we get $x \in P, \alpha(x) \leq r_{2}$ and $\beta(x) \leq L_{2}$. Then

$$
0 \leq x(n) \leq r_{2}, n \in[0, N+2] ;|\Delta x(n)| \leq L_{2} \text { for all } n \in[0, N+1]
$$

$\left(A_{3}\right)$ implies that $f(n, x(n+1), \Delta x(n), \Delta x(n+1)) \leq \min \left\{M_{2}, N_{2}\right\}$.

Since $A_{x} \in\left[0, \sum_{i=0}^{N} f(i, x(i+1), \Delta x(i), \Delta x(i+1))\right]$, given by (10) in the proof of Lemma 2.6, and $\left(A_{3}\right)$ holds, we get

$$
\begin{aligned}
& \alpha(T x) \\
& =\max _{n \in[0, N+2]}|T x(n)| \\
& =\max _{n \in[0, N+2]}\left(B_{x}+\sum_{i=0}^{n-1} \phi^{-1}\left(A_{x}-\sum_{j=0}^{i-1} f(j, x(j+1), \Delta x(j), \Delta x(j+1))\right)\right) \\
& \leq \max _{n \in[0, N+2]}\left[\frac{1}{1-\sum_{i=1}^{m} \alpha_{i}} \sum_{j=1}^{m} \alpha_{j} \sum_{i=0}^{n_{j}-1} \phi^{-1}\left(A_{x}-\sum_{s=0}^{i-1} f(s, x(s+1), \Delta x(s), \Delta x(s+1))\right)\right. \\
& \left.+\frac{A}{1-\sum_{i=1}^{m} \alpha_{i}}+\sum_{i=0}^{n-1} \phi^{-1}\left(A_{x}-\sum_{j=0}^{i-1} f(j, x(j+1), \Delta x(j), \Delta x(j+1))\right)\right] \\
& \leq \max _{n \in[0, N+2]}\left(\frac { 1 } { 1 - \sum _ { i = 1 } ^ { m } \alpha _ { i } } \sum _ { j = 1 } ^ { m } \alpha _ { j } \sum _ { i = 0 } ^ { n _ { j } - 1 } \phi ^ { - 1 } \left(\sum_{s=0}^{N} f(s, x(s+1), \Delta x(s), \Delta x(s+1))\right.\right. \\
& \left.-\sum_{s=0}^{i-1} f(s, x(s+1), \Delta x(s), \Delta x(s+1))\right)+\frac{A}{1-\sum_{i=1}^{m} \alpha_{i}} \\
& \left.+\sum_{i=0}^{n-1} \phi^{-1}\left(\sum_{j=0}^{N} f(j, x(j+1), \Delta x(j), \Delta x(j+1))-\sum_{j=0}^{i-1} f(j, x(j+1), \Delta x(j), \Delta x(j+1))\right)\right) \\
& =\max _{n \in[0, N+2]}\left(\frac{1}{1-\sum_{i=1}^{m} \alpha_{i}} \sum_{j=1}^{m} \alpha_{j} \sum_{i=0}^{n_{j}-1} \phi^{-1}\left(\sum_{s=i}^{N} f(s, x(s+1), \Delta x(s), \Delta x(s+1))\right)\right. \\
& \left.+\frac{A}{1-\sum_{i=1}^{m} \alpha_{i}}+\sum_{i=0}^{n-1} \phi^{-1}\left(\sum_{j=i}^{N} f(j, x(j+1), \Delta x(j), \Delta x(j+1))\right)\right)
\end{aligned}
$$




$$
\begin{aligned}
\leq & \max _{n \in[0, N+2]}\left(\frac{1}{1-\sum_{i=1}^{m} \alpha_{i}} \sum_{j=1}^{m} \alpha_{j} \sum_{i=0}^{n_{j}-1} \phi^{-1}(N-i+1)+\sum_{i=0}^{n-1} \phi^{-1}(N-i+1)\right) \phi^{-1}\left(M_{2}\right) \\
& +\frac{A}{1-\sum_{i=1}^{m} \alpha_{i}} \\
= & \left(\frac{1}{1-\sum_{i=1}^{m} \alpha_{i}} \sum_{j=1}^{m} \alpha_{j} \sum_{i=0}^{n_{j}-1} \phi^{-1}(N-i+1)+\sum_{i=0}^{N+1} \phi^{-1}(N-i+1)\right) \phi^{-1}\left(M_{2}\right) \\
& +\frac{A}{1-\sum_{i=1}^{m} \alpha_{i}} \\
= & r_{2},
\end{aligned}
$$

and

$$
\begin{aligned}
\beta(T x)= & \max _{n \in[0, N+1]}|\Delta(T x)(n)| \\
= & \max _{n \in[0, N+1]}\left|\phi^{-1}\left(A_{x}-\sum_{j=0}^{n-1} f(j, x(j+1), \Delta x(j), \Delta x(j+1))\right)\right| \\
\leq & \max _{n \in[0, N+1]} \phi^{-1}\left(\sum_{j=0}^{N} f(j, x(j+1), \Delta x(j), \Delta x(j+1))\right. \\
& \left.\quad+\sum_{j=0}^{n-1} f(j, x(j+1), \Delta x(j), \Delta x(j+1))\right) \\
= & \max _{n \in[0, N+1]} \phi^{-1}\left(2 \sum_{j=0}^{N} f(j, x(j+1), \Delta x(j), \Delta x(j+1))\right) \\
\leq & \phi^{-1}(2 N+2) \phi^{-1}\left(N_{2}\right) \\
= & L_{2} .
\end{aligned}
$$

So $T x \in \bar{P}\left(\alpha, r_{2} ; \beta, L_{2}\right)$. It follows that $T \bar{P}\left(\alpha, r_{2} ; \beta, L_{2}\right) \subseteq \bar{P}\left(\alpha, r_{2} ; \beta, L_{2}\right)$. These completes Step 1.

Step 2. Prove that $\left(C_{2}\right)$ in Lemma 2.1 holds. For each $y \in \bar{P}\left(\alpha, r_{1} ; \beta, L_{1}\right)$, we prove that $\alpha(T y)<r_{1}$ and $\beta(T y)<L_{1}$ by using $\left(A_{1}\right)$. The proof is similar to above discussion and is omitted.

Step 3. Prove that $\left(C_{1}\right)$ in Lemma 2.1 holds. We prove that

$$
\left\{y \in \bar{P}\left(\alpha, d ; \beta, L_{2} ; \psi, b\right) \mid \psi(y)>b\right\} \neq \emptyset \text { and } \psi(T y)>b
$$

for every $y \in \bar{P}\left(\alpha, d ; \beta, L_{2} ; \psi, b\right)$;

Choose $x(n)=b /\left(2 \sigma_{0}\right)=d / 2$ for all $n \in[0, N+2]$. It is easy to see that

$$
\alpha(x)=\frac{b}{2 \sigma_{0}}<d, \beta(x)=0 \leq L_{2}, \psi(x)=\frac{b}{2 \sigma_{0}}>b
$$


since $\sigma_{0}=\min \left\{\frac{k}{N+2}, \frac{N+2-k}{N+2}\right\}<\frac{1}{2}$.

Then $x \in \bar{P}\left(\alpha, r_{2} ; \beta, L_{2} ; \psi, b\right)$ and $\psi(T x)>b$. Hence

$$
\left\{y \in \bar{P}\left(\alpha, r_{2} ; \beta, L_{2} ; \psi, b\right) \mid \psi(y)>b\right\} \neq \emptyset .
$$

For $x \in \bar{P}\left(\alpha, d ; \beta, L_{2} ; \psi, b\right)$, one sees that

$$
|\Delta x(n)| \leq L_{2}, n \in[0, N+1] ; \quad b \leq x(n) \leq \frac{b}{\sigma_{0}}, n \in[k, N+2-k] .
$$

It follows from $\left(A_{2}\right)$ that

$$
f(n, x(n+1), \Delta x(n), \Delta x(n+1))>M_{2}, n \in[k, N+2-k] .
$$

Similarly to Lemma 2.8 that there exists $n_{0} \in[k, N-k]$ such that $\Delta x\left(n_{0}\right) \geq 0$ and $\Delta x\left(n_{0}+1\right) \leq 0$ and

$$
\begin{aligned}
\max _{n \in[0, N+2]}|(T x)(n)| & =\max _{n \in[0, N+2]}(T x)(n)=(T x)\left(n_{0}+1\right) \\
& \geq \max \left\{(T x)\left(n_{0}\right),(T x)\left(n_{0}+2\right)\right\},
\end{aligned}
$$

we get from $(7),\left(H_{1}\right)$ and $\left(H_{2}\right)$, Lemma 2.8 that

$$
\begin{aligned}
\psi(T x) & =\min _{n \in[k, N+2-k]}|(T x)(n)| \geq \sigma_{0} \max _{n \in[0, N+2]}|(T x)(n)| \geq \sigma_{0}(T x)\left(n_{0}\right) \\
& =\sigma_{0} \sum_{i=0}^{n_{0}-1} \phi^{-1}\left(\sum_{j=i}^{n_{0}-1} f(j, x(j+1), \Delta x(j), \Delta x(j+1))\right) .
\end{aligned}
$$

Similarly, we get

$$
\begin{aligned}
\psi(T x) & =\min _{n \in[k, N+2-k]}|(T x)(n)| \geq \sigma_{0} \max _{n \in[0, N+2]}|(T x)(n)| \geq \sigma_{0}(T x)\left(n_{0}+2\right) \\
& =\sigma_{0} \sum_{i=n_{0}+2}^{N+2} \phi^{-1}\left(\sum_{j=n_{0}+1}^{i-1} f(j, x(j+1), \Delta x(j), \Delta x(j+1))\right) .
\end{aligned}
$$

Then

$$
\begin{array}{r}
\psi(T x) \geq \sigma_{0} \max \left\{\sum _ { i = n _ { 0 } + 2 } ^ { N + 2 } \phi ^ { - 1 } \left(\sum_{j=n_{0}+1}^{i-1} f(j, x(j+1), \Delta x(j), \Delta x(j+1)),\right.\right. \\
\geq \sigma_{0} \max \left\{\sum_{i=n_{0}+2}^{n_{0}-1} \phi^{-1}\left(\sum_{j=i}^{n_{0}-1} f(j, x(j+1), \Delta x(j), \Delta x(j+1))\right)\right\} \\
\sum_{i=k}^{n_{0}-1} \phi^{-1}\left(\sum_{j=n_{0}+1}^{n_{0}-1} f(j, x(j+1), \Delta x(j), \Delta x(j+1))\right\},
\end{array}
$$




$$
\begin{aligned}
& \geq \sigma_{0} \max \left\{\sum_{i=[(N+2) / 2]+1}^{N+2-k} \phi^{-1}\left(\sum_{j=[(N+2) / 2]+1}^{i-1} f(j, x(j+1), \Delta x(j), \Delta x(j+1))\right),\right. \\
& \left.\sum_{i=k}^{[(N+2) / 2]} \phi^{-1}\left(\sum_{j=i}^{[(N+2) / 2]} f(j, x(j+1), \Delta x(j), \Delta x(j+1))\right)\right\} \\
& >\sigma_{0} \max \left\{\sum_{i=[(N+2) / 2]+1}^{N+2-k} \phi^{-1}(i-[(N+2) / 2]-1),\right. \\
& \left.\sum_{i=k}^{[(N+2) / 2]} \phi^{-1}([(N+2) / 2]-i+1)\right\} \phi^{-1}\left(M_{3}\right) \\
& =b .
\end{aligned}
$$

We get $\psi(T x)>b$. Step 3 is complete.

Step 4. Prove that $\left(C_{3}\right)$ in Lemma 2.1 holds. We prove that $\psi(T y)>b$ for $y \in \bar{P}\left(\alpha, r_{2} ; \beta, L_{2} ; \psi, b\right)$ with $\alpha(T y)>\frac{b}{\sigma_{0}}$.

Suppose $x \in \bar{P}\left(\alpha, r_{2} ; \beta, L_{2} ; \psi, b\right)$ with $\alpha(T x)>\frac{b}{\sigma_{0}}$. We get

$$
\psi(T x)=\min _{n \in[k, N+2-k]}|(T x)(n)| \geq \sigma_{0} \max _{n \in[0, N+2]}|(T x)(n)|=\sigma_{0} \alpha(T x)>\sigma_{0} \frac{b}{\sigma_{0}}=b .
$$

This complete Step 4.

It follows from Lemma 2.1 that $\operatorname{BVP}(3)$ has at least three positive solutions $x_{1}, x_{2}, x_{3}$ satisfying

$$
\begin{aligned}
& x_{1} \in P\left(\alpha, r_{1} ; \beta, L_{1}\right), \\
& x_{2} \in\left\{x \in \bar{P}\left(\alpha, r_{2} ; \beta, L_{2} ; \psi, b\right), \psi(x)>b\right\}, \\
& x_{3} \in \bar{P}\left(\alpha, r_{2} ; \beta, L_{2}\right) \backslash\left[\bar{P}\left(\alpha, r_{2} ; \beta, L_{2} ; \psi, b\right) \sqcup \bar{P}\left(\alpha, r_{1} ; \beta, L_{1}\right)\right] .
\end{aligned}
$$

Remark. Consider $\operatorname{BVP}(3)$, suppose that

$\left(H_{3}\right)^{\prime} \frac{1-\sum_{i=1}^{m} \beta_{i}}{1-\sum_{i=1}^{m} \alpha_{i}} A-B<0$, there exists a sequence $q$ such that $f(n, x, u, v) \geq$ $q(n)$ for all $n \in[0, N], x, u, v \in R$ and

$$
\begin{aligned}
& \frac{1-\sum_{i=1}^{m} \beta_{i}}{1-\sum_{i=1}^{m} \alpha_{i}}\left[\left(1-\sum_{i=1}^{m} \alpha_{i}\right) \sum_{i=0}^{N+1} \phi^{-1}\left(\sum_{s=i}^{N} q(s)\right)+\sum_{i=1}^{m} \alpha_{i} \sum_{j=0}^{i-1} \phi^{-1}\left(\sum_{s=j}^{N} q(s)\right)\right] \\
& +\sum_{i=1}^{m} \beta_{i} \sum_{j=n_{i}}^{N+1} \phi^{-1}\left(\sum_{s=j}^{N} q(s)\right)+\frac{1-\sum_{i=1}^{m} \beta_{i}}{1-\sum_{i=1}^{m} \alpha_{i}} A-B>0
\end{aligned}
$$

A similar existence result to Theorem L can be obtained, we omit the details. 


\section{An example}

In this section, we present an example to illustrate the main results.

Example 3.1. Consider the following BVP

(13) $\left\{\begin{array}{l}\Delta\left(|\Delta x(n)|^{2} \Delta x(n)\right)+f(n, x(n+1), \Delta x(n), \Delta x(n+1))=0,0 \leq n \leq 100, \\ x(0)-\frac{1}{2} x(20)=2, \\ x(102)-\frac{1}{3} x(20)=\frac{4}{3},\end{array}\right.$

where $f(n, x, y, w)$ is continuous and positive for all $(n, x, y, w) \in[0, N] \times$ $[0,+\infty) \times \mathbb{R}^{2}$.

Suppose that

(S) there exists a positive number $q$ such that $f(n, x, u, v) \geq q$ for all $n \in$ $[0,100], x \geq 0, u, v \in \mathbb{R}$ and

$$
\phi^{-1}(q)\left[4\left(\frac{1}{2} \sum_{j=0}^{19} \phi^{-1}(j)\right)+2 \sum_{i=0}^{101} \phi^{-1}(i)+\sum_{j=20}^{101} \phi^{-1}(j)\right]>4 .
$$

Corresponding to $\operatorname{BVP}(3)$, we have $N=100, m=1, n_{1}=20, \alpha_{1}=1 / 2$, $\beta_{1}=1 / 3, A=2, B=\frac{4}{3}, \phi(x)=|x|^{2} x$ with $\phi^{-1}(x)=|x|^{-2 / 3} x$. It is easy to see that $\left(H_{1}\right),\left(H_{2}\right)$ hold and $\left(H_{3}\right)$ hold if $(S)$ is valid.

Choose the constant $k=4$, then $\sigma_{0}=\min \left\{\frac{4}{102}, \frac{98}{102}\right\}=\frac{4}{102}, r_{1}=100$, $r_{2}=540000, L_{1}=100, L_{2}=20000$ and $b=180$, then

$$
L_{2}>L_{1}, \quad r_{2}>b / \sigma_{0}>b>r_{1}>\frac{A}{1-\sum_{i=1}^{m} \alpha_{i}} .
$$

One sees that

$$
\begin{aligned}
Q & =\max _{n \in[0,102]}\left(\sum_{i=0}^{19} \phi^{-1}(101-i)^{-2 / 3}+\sum_{i=0}^{n-1} \phi^{-1}(101-i)\right) \\
& =\sum_{i=0}^{19}(101-i)^{-2 / 3}+\sum_{i=0}^{101}(101-i)^{-2 / 3} \\
M_{2} & =\frac{r_{2}^{3}}{Q^{3}}, \quad N_{2}=\frac{L_{2}^{3}}{202}, \quad M_{1}=\frac{r_{1}^{3}}{Q^{3}}, \quad N_{1}=\frac{L_{2}^{3}}{202}, \\
O & =\sigma_{0} \min \left\{\sum_{i=52}^{98}(i-52)^{-2 / 3}, \sum_{i=4}^{51}(52-i)^{-2 / 3}\right\}, \quad M_{3}=\frac{b^{3}}{O^{3}} .
\end{aligned}
$$

If $q<M_{3}<\min \left\{M_{2}, N_{2}\right\}, q<\min \left\{M_{1}, N_{1}\right\}$ and

$f(t, u, v, w)<\min \left\{M_{1}, N_{1}\right\}$ for all $t \in[0,100], u \in[0,100], v, w \in[-100,100]$;

$f(t, u, v, w)>M_{3}$ for all $t \in[4,98], u \in[1800,450 \times 102], v, w \in[-20000,20000]$;

$f(t, u, v, w) \leq \min \left\{M_{2}, N_{2}\right\}$ for all $t \in[0,100], u \in[0,540000]$,

$$
v, w \in[-20000,20000] \text {, }
$$


then Theorem L implies that BVP(13) has at least three positive solutions such that

(i) $x_{1}$ satisfies $\max _{n \in[0,102]} x_{1}(n)<100, \max _{n \in[0,101]}\left|\Delta x_{1}(n)\right|<100$;

(ii) $x_{2}$ satisfies $180<\min _{t \in[4,98]} x_{2}(n)<\max _{n \in[0,102]} x_{2}(n)<540000$, $\max _{n \in[0,101]}\left|\Delta x_{2}(n)\right|<20000$;

(iii) $x_{3}$ satisfies $\max _{n \in[0,102]} x(n) \leq 540000, \max _{n \in[0,101]}|\Delta x(n)| \leq 20000$, $\min _{t \in[4,98]} x_{3}(n)<180, \max _{n \in[0,102]} x_{3}(n)>100$, and $\max _{n \in[0,102]}\left|\Delta x_{3}(n)\right|$ $>100$.

Acknowledgement. The author is thankful to the editors for taking much time and effort to check the paper and rearrange the order of the reference list.

\section{References}

[1] D. Anderson, Discrete third-order three-point right-focal boundary value problems, Advances in difference equations, IV. Comput. Math. Appl. 45 (2003), no. 6-9, 861-871.

[2] D. Anderson and R. I. Avery, Multiple positive solutions to a third-order discrete focal boundary value problem, Comput. Math. Appl. 42 (2001), no. 3-5, 333-340.

[3] R. I. Avery and A. C. Peterson, Three positive fixed points of nonlinear operators on ordered Banach spaces, Comput. Math. Appl. 42 (2001), no. 3-5, 313-322.

[4] N. Aykut, Existence of positive solutions for boundary value problems of second-order functional difference equations, Comput. Math. Appl. 48 (2004), no. 3-4, 517-527.

[5] Z. Bai and W. Ge, Existence of three positive solutions for a one-dimensional pLaplacian, Acta Math. Sinica (Chin. Ser.) 49 (2006), no. 5, 1045-1052.

[6] X. Cai and J. Yu, Existence theorems for second-order discrete boundary value problems, J. Math. Anal. Appl. 320 (2006), no. 2, 649-661.

[7] W. Cheung, J. Ren, P. J. Y. Wong, and D. Zhao, Multiple positive solutions for discrete nonlocal boundary value problems, J. Math. Anal. Appl. 330 (2007), no. 2, 900-915.

[8] J. R. Graef and J. Henderson, Double solutions of boundary value problems for $2 \mathrm{mth}$ order differential equations and difference equations, Comput. Math. Appl. 45 (2003), no. 6-9, 873-885.

[9] Z. He, On the existence of positive solutions of p-Laplacian difference equations, J. Comput. Appl. Math. 161 (2003), no. 1, 193-201.

[10] I. Y. Karaca, Discrete third-order three-point boundary value problem, J. Comput. Appl. Math. 205 (2007), no. 1, 458-468.

[11] R. Leggett and L. Williams, Multiple positive fixed points of nonlinear operators on ordered Banach spaces, Indiana Univ. Math. J. 28 (1979), no. 4, 673-688.

[12] Y. $\mathrm{Li}$ and $\mathrm{L}$. Lu, Existence of positive solutions of p-Laplacian difference equations, Appl. Math. Lett. 19 (2006), no. 10, 1019-1023.

[13] H. Pang, H. Feng, and W. Ge, Multiple positive solutions of quasi-linear boundary value problems for finite difference equations, Appl. Math. Comput. 197 (2008), no. 1, 451456.

[14] P. J. Y. Wong and R. P. Agarwal, Existence theorems for a system of difference equations with (n,p)-type conditions, Appl. Math. Comput. 123 (2001), no. 3, 389-407.

[15] P. J. Y. Wong and L. Xie, Three symmetric solutions of Lidstone boundary value problems for difference and partial difference equations, Comput. Math. Appl. 45 (2003), no. $6-9,1445-1460$.

[16] C. Yang and P. Weng, Green functions and positive solutions for boundary value problems of third-order difference equations, Comput. Math. Appl. 54 (2007), no. 4, 567-578.

[17] J. Yu and Z. Guo, Boundary value problems of discrete generalized Emden-Fowler equation, Sci. China Ser. A 49 (2006), no. 10, 1303-1314. 
[18] G. Zhang and R. Medina, Three-point boundary value problems for difference equations, Comput. Math. Appl. 48 (2004), no. 12, 1791-1799.

Department of Mathematics

Guangdong University of Business Studies

Guangzhou 510320, P. R. China

E-mail address: liuyuji888@sohu.com 